\title{
Phthalic Acid Esters: Natural Sources and Biological Activities
}

\author{
Ling Huang ${ }^{1,2,+}$, Xunzhi Zhu ${ }^{3,+}$, Shixing Zhou ${ }^{1,4}$, Zhenrui Cheng ${ }^{1}$, Kai Shi ${ }^{1,4}$, Chi Zhang ${ }^{5, * \mathbb{D}}$ \\ and Hua Shao $1,2,4, *$ (D)
}

check for updates

Citation: Huang, L.; Zhu, X.; Zhou, S.; Cheng, Z.; Shi, K.; Zhang, C.; Shao, H. Phthalic Acid Esters: Natural Sources and Biological Activities. Toxins 2021, 13, 495. https://doi.org/ $10.3390 /$ toxins 13070495

Received: 13 June 2021

Accepted: 14 July 2021

Published: 16 July 2021

Publisher's Note: MDPI stays neutral with regard to jurisdictional claims in published maps and institutional affiliations.

\section{Copyright: (c) 2021 by the authors.} Licensee MDPI, Basel, Switzerland. This article is an open access article distributed under the terms and conditions of the Creative Commons Attribution (CC BY) license (https:// creativecommons.org/licenses/by/ $4.0 /)$.
1 State Key Laboratory of Desert and Oasis Ecology, Xinjiang Institute of Ecology and Geography, Chinese Academy of Sciences, Urumqi 830011, China; huangling201@mails.ucas.ac.cn (L.H.); zhoushixing16@mails.ucas.ac.cn (S.Z.); czr24czr@163.com (Z.C.); shikai19@mails.ucas.ac.cn (K.S.)

2 Research Center for Ecology and Environment of Central Asia, Xinjiang Institute of Ecology and Geography, Chinese Academy of Sciences, Urumqi 830011, China

3 Institute of Botany, Jiangsu Province and Chinese Academy of Sciences, Nanjing 210014, China; zhuxunzhi@cnbg.net

4 University of Chinese Academy of Sciences, Beijing 100049, China

5 Shandong Provincial Key Laboratory of Water and Soil Conservation and Environmental Protection, College of Resources and Environment, Linyi University, Linyi 276000, China

* Correspondence: zc@ms.xjb.ac.cn (C.Z.); shaohua@ms.xjb.ac.cn (H.S.)

+ These authors contributed equally to this work.

\begin{abstract}
Phthalic acid esters (PAEs) are a class of lipophilic chemicals widely used as plasticizers and additives to improve various products' mechanical extensibility and flexibility. At present, synthesized PAEs, which are considered to cause potential hazards to ecosystem functioning and public health, have been easily detected in the atmosphere, water, soil, and sediments; PAEs are also frequently discovered in plant and microorganism sources, suggesting the possibility that they might be biosynthesized in nature. In this review, we summarize that PAEs have not only been identified in the organic solvent extracts, root exudates, and essential oils of a large number of different plant species, but also isolated and purified from various algae, bacteria, and fungi. Dominant PAEs identified from natural sources generally include di- $n$-butyl phthalate, diethyl phthalate, dimethyl phthalate, di(2-ethylhexyl) phthalate, diisobutyl phthalate, diisooctyl phthalate, etc. Further studies reveal that PAEs can be biosynthesized by at least several algae. PAEs are reported to possess allelopathic, antimicrobial, insecticidal, and other biological activities, which might enhance the competitiveness of plants, algae, and microorganisms to better accommodate biotic and abiotic stress. These findings suggest that PAEs should not be treated solely as a "human-made pollutant" simply because they have been extensively synthesized and utilized; on the other hand, synthesized PAEs entering the ecosystem might disrupt the metabolic process of certain plant, algal, and microbial communities. Therefore, further studies are required to elucidate the relevant mechanisms and ecological consequences.
\end{abstract}

Keywords: phthalic acid esters; natural sources; biological activity; di- $n$-butyl phthalate; di(2ethylhexyl) phthalate

Key Contribution: PAEs detected in the environment are mostly considered human-made pollutants; however, our review provides evidence that they can actually be biosynthesized by certain species of plants, algae, bacteria, fungi, etc., to enhance the competitiveness of their hosts. Their biological activities have the potential to be explored and utilized further.

\section{Introduction}

Phthalic acid esters (PAEs) are common plasticizers added to polymeric materials to improve their flexibility and workability [1]. PAEs have been widely used in numerous consumer products, including cosmetics, food packaging, building materials, medical supplies, home furnishings, etc., due to their characteristic properties, such as their good insulation, 
high strength, excellent corrosion resistance, low cost, and ease of fabrication [2-4]. The current global production of PAEs is estimated at 300 million tons, and it is expected to reach 500 million tons by 2050, most of which will be single-use products [5]. Moreover, China has become the world's largest producer, consumer, and importer of plasticizers, accounting for nearly $42 \%$ of the world's consumption in 2017 [6]. As one of the most abundantly produced phthalates, di(2-ethylhexyl) phthalate accounts for one-third and $80 \%$ of the phthalates made in the European Union and China, respectively [7]. With such extensive application of phthalate-containing products, PAEs have attracted increasing attention as environmental and biomedical pollutants, which may invisibly enter the human body through airborne transmission, skin contact, and food chain transmission, constituting potential health and ecological system threats [8]. In fact, a number of studies have been carried out to investigate the toxicity of PAEs on human beings and/or animals. Epidemiologic studies found that early phthalates exposure could induce significant neurodevelopmental damage [9]. Some PAEs have been proven to possess reproductive and developmental toxicities to animals and are suspected of causing endocrine-disrupting effects to humans [10-12]. PAEs were also harmful to aquatic organisms. Di- $n$-butyl, diethyl phthalate, and their mixture were found to effectively activate zebrafish embryos' antioxidant system and lead to immunotoxicity and neurotoxicity [13,14]. Zhao et al. (2014) reported that di-n-butyl and di(2-ethylhexyl) phthalate disrupted the antioxidant system of carps, meanwhile combined exposure to these two compounds exacerbated this change [15].

Up to now, most of the published literature has focused on the detection methods, pollution distribution, and toxicological hazards of PAEs. However, the natural sources of various PAEs are rarely studied. The first report of phthalic acid as a natural substance was conducted by Schmid and Karrer (1945) [16] and, since then, more than 50 different derivatives of PAEs have been reported from different taxonomic groups, including bacteria, actinomycetes, fungi, fern, higher plants, and even animals [17]. What remains unclear, however, is that in many cases, it is rather complicated to determine whether these compounds come from synthesized materials that later cause contamination of the air, water, or soil, or whether they may be produced by the plants and microorganisms themselves. The objective of this review is to summarize the plant and microorganism origin of PAEs so as to better understand their possible sources: Are they synthesized chemicals, or are they naturally occurring secondary metabolites?

\section{Physicochemical Properties and Applications of PAEs}

Phthalic acid esters (dialkyl or alkyl aryl esters of 1,2-benzenedicarboxylic acid), usually called PAEs, phthalate esters, or just phthalates, are a group of important derivatives of phthalic acids which are synthesized from phthalic anhydride and specific alcohols by Fischer esterification $[18,19]$. PAEs based on hydrogen bond and van der Waals force interconnection are hydrophobic compounds with $\log K_{\text {ow }}$ ranging from 1.6 to 12 [20]. Most of the phthalate esters are colorless liquids with a low volatility, high boiling point, and poor solubility in water, but they are soluble in organic solvents and oils [8]. These esters' general chemical structure consists of a rigid planar aromatic ring and two malleable nonlinear fatty side chains. The two side-chain groups can be the same or not, and there are approximately 30 types of different side chains, ranging from dimethyl phthalate to tridecyl ester [21]. Due to phthalate esters being widespread in the biosphere and potential hazards in relation to ecosystem functioning and public health, six PAEs have been listed as priority pollutants by the United States Environmental Protection Agency and the European Union [20,22], including dimethyl phthalate, diethyl phthalate, di- $n$-butyl phthalate, butyl benzyl phthalate, di(2-ethylhexyl) phthalate, and di- $n$-octyl phthalate. These phthalate esters' physicochemical properties and common applications are summarized in Table 1 and Figure 1. 


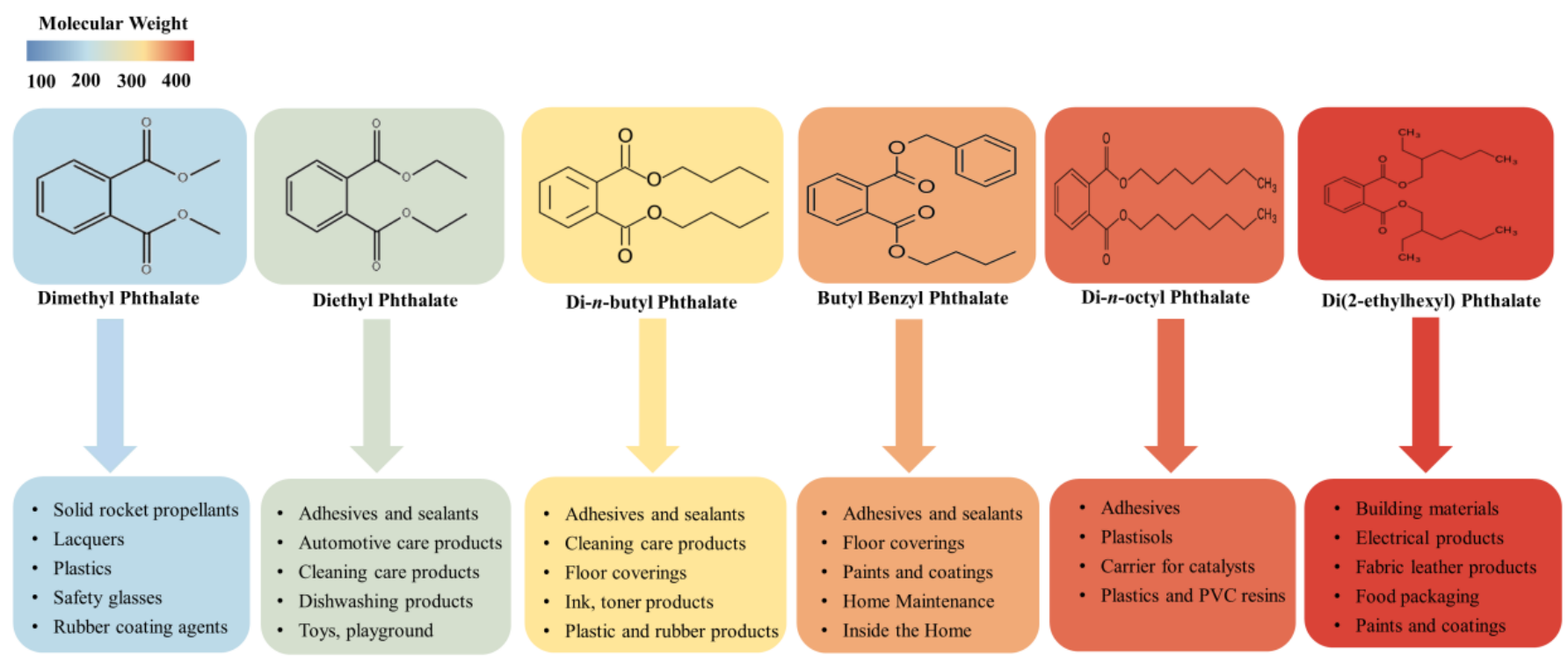

Figure 1. The application of six PAEs listed as priority pollutants.

Table 1. Physicochemical properties and application of six PAEs listed as priority pollutants.

\begin{tabular}{ccccccccc}
\hline PAEs & $\begin{array}{c}\text { Molecular } \\
\text { Formula }\end{array}$ & $\begin{array}{c}\text { Molecular } \\
\text { Weight }\end{array}$ & $\begin{array}{c}\text { CAS Reg- } \\
\text { istration } \\
\text { Number }\end{array}$ & $\begin{array}{c}\text { Specific } \\
\text { Gravity } \\
\left(\mathbf{2 0}{ }^{\circ} \mathbf{C}\right)\end{array}$ & $\begin{array}{c}\text { Water } \\
\text { Solubility } \\
\text { (mg/L) }\end{array}$ & $\begin{array}{c}\text { log } \\
\boldsymbol{K}_{\text {ow }}\end{array}$ & $\begin{array}{c}\text { Melting } \\
\text { Point } \\
\left({ }^{\circ} \mathbf{C}\right)\end{array}$ & Application \\
\hline $\begin{array}{c}\text { Dimethyl } \\
\text { phthalate }\end{array}$ & $\mathrm{C}_{10} \mathrm{H}_{10} \mathrm{O}_{4}$ & 194.18 & $131-11-3$ & 1.19 & 4000 & 1.47 & 5.5 & $\begin{array}{c}\text { Insect repellent, personal } \\
\text { care products, etc. }\end{array}$ \\
\hline $\begin{array}{c}\text { Diethyl } \\
\text { phthalate }\end{array}$ & $\mathrm{C}_{12} \mathrm{H}_{14} \mathrm{O}_{4}$ & 222.24 & $84-66-2$ & 1.12 & 1000 & 2.38 & -40 & $\begin{array}{c}\text { Personal care products, } \\
\text { plasticizers, cosmetics, etc. }\end{array}$ \\
\hline $\begin{array}{c}\text { Di- } n \text {-butyl } \\
\text { phthalate }\end{array}$ & $\mathrm{C}_{14} \mathrm{H}_{38} \mathrm{O}_{4}$ & 278.35 & $84-74-2$ & 1.05 & 11.2 & 3.74 & -35 & $\begin{array}{c}\text { PVC plastics, explosive } \\
\text { materials, nail paints, etc. }\end{array}$ \\
\hline $\begin{array}{c}\text { Butyl } \\
\text { benzyl } \\
\text { phthalate }\end{array}$ & $\mathrm{C}_{19} \mathrm{H}_{20} \mathrm{O}_{4}$ & 302.39 & $85-68-7$ & 1.11 & 2.7 & 4.59 & -35 & $\begin{array}{c}\text { Rapping materials, food } \\
\text { conveyor belts, artificial } \\
\text { letter, traffic cones, etc. }\end{array}$ \\
\hline $\begin{array}{c}\text { Di(2- } \\
\text { ethylhexyl) } \\
\text { phthalate }\end{array}$ & $\mathrm{C}_{24} \mathrm{H}_{38} \mathrm{O}_{4}$ & 390.62 & $117-81-7$ & 0.99 & 0.003 & 7.5 & -40 & $\begin{array}{c}\text { Medical devices, food } \\
\text { packaging, building } \\
\text { products, children's } \\
\text { products, etc. }\end{array}$ \\
\hline $\begin{array}{c}\text { Di- } n \text {-octyl } \\
\text { phthalate }\end{array}$ & $\mathrm{C}_{24} \mathrm{H}_{38} \mathrm{O}_{4}$ & 390.62 & $117-84-0$ & 0.99 & 0.0005 & 8.06 & -25 & $\begin{array}{c}\text { Conveyor belts, pool liners, } \\
\text { garden hoses, etc. }\end{array}$ \\
\hline
\end{tabular}

PAEs are a class of lipophilic chemicals widely used in the plastics manufacturing industries as plasticizers and additives to improve the mechanical extensibility and flexibility of various products, such as plastics, paints, and synthetic fibers [23]. Phthalates of lower molecular weight, such as dimethyl phthalate, diethyl phthalate, and di- $n$-butyl phthalate, are widely used in cosmetics and personal care products; dimethyl phthalate and diethyl phthalate allow perfume fragrances to evaporate more slowly, making the scent linger longer, and a small amount of di-n-butyl phthalate can make nail polish chip-resistant. Di- $n$-butyl phthalate is also used in cellulose esters, printing inks, latex adhesives, and insect repellents [11,24].

Higher phthalate molecules, such as di(2-ethylhexyl) phthalate, diisononyl phthalate, and butyl benzyl phthalate, have a wide range of applications as plasticizers in the polymer industry to improve flexibility, workability, and general handling properties, and about $80 \%$ of PAEs are used for this purpose $[20,25]$. The stability, fluidity and low volatility of these compounds make them very suitable for manufacturing PVC and other resins, such as polyvinyl acetates and polyurethanes [26]. One of the most widespread phthalate plasticizers, di(2-ethylhexyl) phthalate, has several useful applications in numerous consumer 
products, commodities, and building materials [27]. Diisononyl phthalate is commonly used in garden hoses, pool liners, flooring tiles, tarps, and toys. Additionally, butyl benzyl phthalate, as a component of materials, is extensively used in vinyl flooring, synthetic leather, inks, and adhesives [19]. Phthalates are not covalently bound to the polymer matrix, rather they usually remain present as a freely mobile and leachable phase; therefore, they can be lost from soft plastic over time and released to the environment during production and manufacture. Not surprisingly, phthalates can often be found in freshwater lakes and oceans [28,29], urban and suburban soil [30,31], the atmosphere [32,33], and sediments [34,35]. Bu et al. (2020) [36] summarized the concentrations of six representative phthalates from published papers in the last twenty years (2000-2019) to analyze the pollution characteristics of phthalates worldwide and found that their mean concentration in settled dust was $500.02 \mu \mathrm{g} / \mathrm{g}$ in North America, $580.12 \mu \mathrm{g} / \mathrm{g}$ in Europe, and $945.45 \mu \mathrm{g} / \mathrm{g}$ in Asia, with DEHP being the most predominant phthalate, with mean and median values of $615.78 \mu \mathrm{g} / \mathrm{g}$ and $394.03 \mu \mathrm{g} / \mathrm{g}$, respectively; the mean concentration of six representative phthalates in indoor air was $598.14 \mathrm{ng} / \mathrm{m}^{3}$ in North America, $823.98 \mathrm{ng} / \mathrm{m}^{3}$ in Europe, and $1710.26 \mathrm{ng} / \mathrm{m}^{3}$ in Asia. In another study, Hu et al. (2020) [37] detected 8 PAEs in 67 sediment samples collected from Hangzhou Bay, Taizhou Bay, and Wenzhou Bay in China; the total concentrations of detected PAEs were in the range of 654-2603 ng/g, with di(2-ethylhexyl) phthalate being the predominant PAE (mean $663 \mathrm{ng} / \mathrm{g}$, accounting for a mean of $52 \%$ of total PAEs).

\section{Natural Existence of PAEs in Living Organisms}

\subsection{PAEs from Plant Sources}

Literature surveys revealed that PAEs were previously detected in different parts (stems, leaves, flowers, fruits, roots, and seeds) of 60 plant species that belong to 38 families, as well as in various algae, such as Gracilaria lemaneiformis, Chaetomorpha basiretorsa, and Cladophora fracta (Figure 2). PAEs were often found in the following families: Lamiaceae (seven species, accounting for $11.7 \%$ of the total), Rosaceae (four species, accounting for $6.7 \%$ ), Solanaceae (four species, accounting for 6.7\%), Liliaceae (three species, accounting for $5 \%$ ), and Asteraceaeare (three species, accounting for 5\%) (Table 2), which represented 35\% of the total species, with di- $n$-butyl phthalate, diisobutyl phthalate, and di(2-ethylhexyl) phthalate being the most frequently detected PAEs.

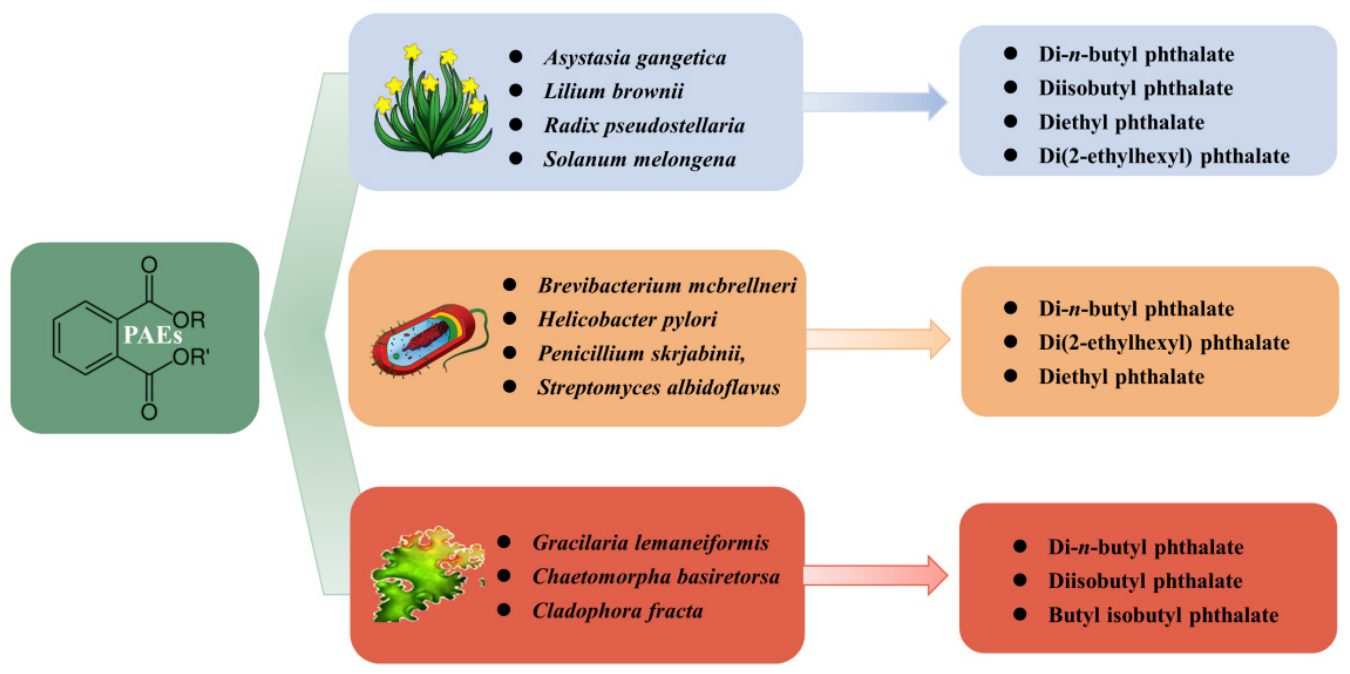

Figure 2. Natural existence of PAEs in living organisms.

PAEs have been detected via GC/MS in the organic extracts of certain plant species, with their percentages varying from $1.0 \%$ to $32.0 \%$ (Table 2). For instance, di- $n$-butyl phthalate was found in the extracts of Brassica oleracea (32.0\%), Ixora amplexicaulis (15.0\%), Gossypium hirsutum (7.9\%), and Zea mays (7.0\%) [42-45]. Di- $n$-octyl phthalate was identified 
to be abundant in the extracts of Prunella vulgaris (29.9\%), Jatropha curcas (21.6\%), and Photinia parvifolia (10.1\%) [46-48]. Other PAEs, such as diethyl phthalate, isobutyl octyl phthalate, etc., were also reported in different plant extracts. Noteworthily, some of the detected PAEs are not commonly used in industry, implying that they might originate from biosynthesis rather than from contaminated soil or air.

Most PAEs were found in plant-derived essential oils (EOs). EOs can be synthesized by all plant organs (flowers, buds, seeds, leaves, twigs, bark, herbs, wood, fruits, and roots), which can be extracted using traditional hydrodistillation, organic solvent-steam distillation, headspace solid-phase microextraction (HS-SPME), and supercritical $\mathrm{CO}_{2}$ fluid extraction $\left(\mathrm{CO}_{2}-\mathrm{SFE}\right)$ procedures [49]. EOs not only play an important role in many physiological and biochemical reactions, but are also widely utilized in pharmaceutical, sanitary, cosmetic, agricultural, and food industries [50,51]. PAEs are constantly being identified in different varieties of Eos. Twenty-six plants have been reported to contain PAEs, with di- $n$-butyl phthalate being the most abundant constituent, which has been found in eighteen species, with the percentage ranging from $1.5 \%$ to $87.2 \%$ (Table 2 ). Species that are rich in di- $n$-butyl phthalate include Radix pseudostellaria $(87.2 \%)$, Clerodendrum inerme (59.3\%), Pyrola rotundifolia (40.5\%), Osmanthus fragrans (15.1\%), and Alocasia macrorrhiza (14.4\%) [52-56]. Di(2-ethylhexyl) phthalate is also a common component detected in Eos; for instance, it is found in the Eos produced by Cirsium japonicum (30.8\%), Pyrus ussriensis (29.4\%), Ziziphus mauritiana (18.0\%), and Clerodendrum inerme (17.3\%) [56-59].

Some PAEs were found in the litter and root exudates of plants, which are actually considered the primary inputs of allelochemicals to the external environment that affect neighboring plants' growth [60]. At least in part, allelopathy helps explain the mechanism of the establishment of dominance of certain plant species, including invasive alien species; allelopathy also provides a theoretical basis for revealing the mechanism of crop intercropping and rotation obstacles in agricultural production $[60,61]$. In fact, some PAEs, such as di- $n$-octyl phthalate, have been confirmed to be active allelochemicals [62]. Di- $n$-butyl phthalate and diisobutyl phthalate are the most frequently identified PAEs in root exudates of plants such as Solanum lycopersicum, Capsicum annuum, Z. mays, Solanum melongena, etc. (Table 2). Cheng and Xu (2012) [63] collected root exudates of Lilium brownii, which revealed that phthalate acid esters, such as diisooctyl phthalate (52.1\%) and di(2-ethylhexyl) phthalate (41.0\%), were dominant. Zhou et al. (2010) [64] studied the root exudates of grafted eggplants using the root soaking method, which led to the identification of di- $n$-butyl phthalate $(13.6 \%)$, diisobutyl phthalate $(1.9 \%)$, and diisononyl phthalate $(0.8 \%)$. GC-MS analysis showed that there were eleven organic compounds in the methanol extract of root exudates of Allium fistulosum, including derivatives of phthalate ester, such as diisooctyl phthalate (52.1\%) and di(2-ethylhexyl) phthalate $(41.0 \%)$.

Although the GC/MS procedure is effective in detecting PAEs, it has its limitations. In some studies, calculation of the retention indices (RI) was ignored; thus, the accuracy of the identification of PAEs was reduced. Traditionally, preparative chromatographic purification of secondary metabolites produced by plants includes the application of silica gel column chromatography, sephadex LH-20 gel column chromatography, semi-preparative HPLC, preparative TLC, etc. During this process, PAEs such as di- $n$-butyl phthalate, diisobutyl phthalate, etc., were purified from different plant species (Table 2). Liu et al. (2011) [65] isolated di-n-butyl phthalate and diisobutyl phthalate from the leaves and stems of Toona ciliata. Shi et al. (2005) [66] obtained di- $n$-butyl phthalate and diisobutyl phthalate from $C$. basiretors $a$ for the first time by spectroscopic methods. As secondary metabolites, di- $n$-butyl phthalate and diisobutyl phthalate were also isolated from the whole plants of C. fracta [67], the root of Croton lachynocarpus [68], and the fruits of Pyrus bretschneideri [69]. Consequently, PAEs identified and purified in plant materials illustrate that the plants could synthesize them to some extent. 
Table 2. PAEs detected in plant materials.

\begin{tabular}{|c|c|c|c|c|c|}
\hline Family & Identified from & Origin & Type of PAEs & $\begin{array}{l}\text { Relative Content } \\
\text { of PAEs (\%) * }\end{array}$ & References \\
\hline \multirow{14}{*}{ Acanthaceae } & \multirow{3}{*}{ Avicennia marina } & \multirow{3}{*}{ Fruits } & Diethyl phthalate & 1.2 & \multirow{3}{*}{ [70] } \\
\hline & & & Dimethyl phthalate & 0.6 & \\
\hline & & & Methyl nonyl phthalate & 0.4 & \\
\hline & \multirow{11}{*}{ Asystasia gangetica } & \multirow{11}{*}{ Aerial Parts } & Diisobutyl phthalate & 6.1 & \multirow{11}{*}{ [71] } \\
\hline & & & Bis-Decyloctyl phthalate & 5.7 & \\
\hline & & & Bis-Diundecyl phthalate & 5.7 & \\
\hline & & & Bis-Decylhexyl phthalate & 4.2 & \\
\hline & & & Bis-isodecylhexyl phthalate & 4.1 & \\
\hline & & & Diheptyl phthalate & 3.6 & \\
\hline & & & Bis-Didecyl phthalate & 2.6 & \\
\hline & & & Bis-Heptyloctyl phthalate & 2.4 & \\
\hline & & & Di-n-butyl phthalate & 2.3 & \\
\hline & & & Di(2-ethylhexyl) phthalate & 1.5 & \\
\hline & & & Bis-7-Methy loctyl phthalate & 1.0 & \\
\hline & & & Bis (2-isobutyl) phthalate & 32.5 & \\
\hline Araceae & Alocasia macrorrhiza & Whole Plants & Di-n-butyl phthalate & 14.4 & [5o] \\
\hline \multirow{8}{*}{ Asteraceae } & Ageratina adenophora & Leaves, Shoots & $\begin{array}{l}\text { Di(2-ethylhexyl) phthalate } \\
\text { Di- } n \text {-butyl phthalate }\end{array}$ & $\mathrm{N} / \mathrm{A}^{* *}$ & {$[72]$} \\
\hline & & & Di(2-ethylhexyl) phthalate & 30.8 & \multirow{6}{*}{ [57] } \\
\hline & & & Diisooctyl phthalate & 16.6 & \\
\hline & & & Mono (2-ethylhexyl) phthalate & 16.0 & \\
\hline & Cirsium japonicum & Whole Plants & Diisobutyl phthalate & 1.1 & \\
\hline & & & Butyloctyl phthalate & 0.7 & \\
\hline & & & Di- $n$-octyl phthalate & 0.1 & \\
\hline & Chrysanthemum indicum & Leaves, Stems & Diethyl phthalate & $\mathrm{N} / \mathrm{A}$ & [73] \\
\hline & & & Di- $n$-butyl phthalate & & \\
\hline Apiaceae & Angelica sinensis & Roots & Di(2-ethylhexyl) phthalate & N/A & [74] \\
\hline & & & Bis (2-methylpropyl) phthalate & & \\
\hline \multirow{4}{*}{ Brassicaceae } & \multirow{4}{*}{ Brassica oleracea } & \multirow{4}{*}{ Stalks } & Di- $n$-butyl phthalate & 32.0 & \multirow{4}{*}[42]{} \\
\hline & & & Diisooctyl phthalate & 18.5 & \\
\hline & & & Diisobutyl phthalate & 3.4 & \\
\hline & & & Diethyl phthalate & 1.3 & \\
\hline \multirow{2}{*}{ Chenopodiaceae } & \multirow{2}{*}{ Beta vulgaris } & \multirow{2}{*}{ Root Exudates } & Di- $n$-butyl phthalate & 47.2 & \multirow{2}{*}{ [75] } \\
\hline & & & Diisobutyl phthalate & 8.6 & \\
\hline \multirow{3}{*}{ Campanulaceae } & \multirow{3}{*}{ Campanula colorata } & \multirow{3}{*}{ Whole Plants } & Butyloctyl phthalate & 10.2 & \\
\hline & & & Di- $n$-butyl phthalate & 7.4 & [76] \\
\hline & & & Diisooctyl phthalate & 0.6 & \\
\hline Calycanthaceae & Chimonanthus praecox & Flowers & Di- $n$-butyl phthalate & 4.5 & [77] \\
\hline & Cladophora fracta & Whole Plants & Diisobutyl phthalate & & {$[67]$} \\
\hline Cladophoraceae & & Whole Plants & Di- $n$-butyl phthalate & $\mathrm{N} / \mathrm{A}$ & {$[0 /]$} \\
\hline & Chaetomorpha basiretorsa & Whole Plants & $\begin{array}{l}\text { Di- } n \text {-butyl phthalate } \\
\text { Diisobutyl phthalate }\end{array}$ & $\mathrm{N} / \mathrm{A}$ & [66] \\
\hline Cyperaceae & Fimbristylis miliacea & Whole Plants & Di- $n$-octyl phthalate & N/A & [62] \\
\hline Crassulaceae & $\begin{array}{l}\text { Hylotelephium } \\
\text { erythrostictum }\end{array}$ & Flowers & Di- $n$-butyl phthalate & 1.2 & [78] \\
\hline Convolvulaceae & Ipomoea carnea & Whole Plants & Di-n-butyl phthalate & $\mathrm{N} / \mathrm{A}$ & [79] \\
\hline & & & Di-n-butyl phthalate & 87.2 & \\
\hline Caryophyllaceae & Radix pseudostellariae & Whole Plants & Ditridecyl phthalate & 0.7 & [52] \\
\hline & & & Di- $n$-butyl phthalate & & \\
\hline & Croton lachynocarpus & Roots & Diisobutyl phthalate & N/A & [68] \\
\hline Euphorbiaceae & & & Butyl isobutyl phthalate & & \\
\hline & Jatropha curcas & Leaves & Di- $n$-octyl phthalate & 21.6 & [46] \\
\hline & Pyrola rotundifolia & Whole Plants & Di- $n$-butyl phthalate & 40.5 & [53] \\
\hline Ericaceae & Rhododendron calonhutum & & Di- $n$-butyl phthalate & 4.9 & {$[80]$} \\
\hline & R nonoa naron calop nytum & Flowers & Diisobutyl phthalate & 1.4 & {$[80]$} \\
\hline
\end{tabular}


Table 2. Cont.

\begin{tabular}{|c|c|c|c|c|c|}
\hline Family & Identified from & Origin & Type of PAEs & $\begin{array}{l}\text { Relative Content } \\
\text { of PAEs (\%)* }\end{array}$ & References \\
\hline \multirow{3}{*}{ Fabaceae } & \multirow{2}{*}{ Dalbergia odorifera } & \multirow{2}{*}{ Flowers } & Di- $n$-butyl phthalate & 14.0 & \multirow{2}{*}{ [81] } \\
\hline & & & Diisooctyl phthalate & 4.4 & \\
\hline & Medicago sativa & Root Exudates & Di-n-butyl phthalate & 10.7 & {$[82]$} \\
\hline Gracilariaceae & Gracilaria lemaneiformis & Whole Plants & Butyl isobutyl phthalate & $\mathrm{N} / \mathrm{A}$ & {$[83]$} \\
\hline Gesneriaceae & Lysionotus pauciflorus & Whole Plants & Diisobutyl phthalate & 2.7 & [84] \\
\hline Hypericaceae & Hypericum scabrum & Seeds, Leaves & Di(2-ethylhexyl) phthalate & 5.8 & [85] \\
\hline \multirow{14}{*}{ Liliaceae } & \multirow{6}{*}{ Allium fistulosum } & \multirow{6}{*}{ Root Exudates } & Diisooctyl phthalate & 11.4 & \multirow{6}{*}[86]{} \\
\hline & & & Di- $n$-butyl phthalate & 4.7 & \\
\hline & & & Diethyl phthalate & 3.2 & \\
\hline & & & Dimethyl phthalate & 0.9 & \\
\hline & & & Diisobutyl phthalate & 0.7 & \\
\hline & & & Butyl methyl phthalate & 0.6 & \\
\hline & \multirow{5}{*}{ Lilium brownii } & \multirow{5}{*}{ Root Exudates } & Diisooctyl phthalate & 52.1 & \multirow{5}{*}[63]{} \\
\hline & & & Di(2-ethylhexyl) phthalate & 41.0 & \\
\hline & & & Methyl 2-ethylhexyl phthalate & 0.9 & \\
\hline & & & 2-ethyl hexyl butyl phthalate & 0.8 & \\
\hline & & & Di- $n$-butyl phthalate & 0.3 & \\
\hline & \multirow{3}{*}{ Paris polyphylla } & \multirow{3}{*}{ Roots } & Isobutyl-3-pentenyl phthalate & 24.7 & \multirow{3}{*}[87]{} \\
\hline & & & Butyl-2-isobutyl phthalate & 5.5 & \\
\hline & & & Di(2-ethylhexyl) phthalate & 4.2 & \\
\hline \multirow{14}{*}{ Lamiaceae } & \multirow{2}{*}{ Clerodendrum inerme } & \multirow{2}{*}{ Leaves } & Di- $n$-butyl phthalate & 59.3 & \multirow{2}{*}[56]{} \\
\hline & & & Di(2-ethylhexyl) phthalate & 17.3 & \\
\hline & \multirow{2}{*}{ Melissa officinalis } & & Diisobutyl phthalate & 2.5 & \\
\hline & & Aerial Parts & Di-n-butyl phthalate & 1.4 & [88] \\
\hline & & & 2-ethylhexyl undecyl phthalate & 5.3 & \\
\hline & Ocimum obovatum & Leaves & Di- $n$-butyl phthalate & 4.5 & [89] \\
\hline & & & Diisobutyl phthalate & 13.4 & \\
\hline & Phlomis umbrosa & Flowers & Di-n-butyl phthalate & 1.5 & [90] \\
\hline & & & Butyl isobutyl phthalate & 0.4 & \\
\hline & Prunella zuloaris & Whole Plants & Di-n-octyl phthalate & 29.9 & {$[47]$} \\
\hline & Prunetla outgarts & Whole Plants & Diethyl phthalate & 2.5 & [4/] \\
\hline & Phlomis medicinalis & Roots & Butyl isobutyl phthalate & $\mathrm{N} / \mathrm{A}$ & [91] \\
\hline & & & Di-n-butyl phthalate & 8.3 & \\
\hline & Scutellaria barbata & Whole Plants & Diisobutyl phthalate & 3.6 & [92] \\
\hline Malvaceae & Gossypium hirsutum & Stalks & Di-n-butyl phthalate & 7.9 & {$[45]$} \\
\hline & & & $\begin{array}{c}\text { Phthalic acid, hex-3-yl isobutyl } \\
\text { ester }\end{array}$ & 9.7 & \\
\hline Myricaceae & Myricarubra sieb & Fruits & Diisooctyl phthalate & 4.2 & [93] \\
\hline & & & Di-n-butyl phthalate & 2.0 & \\
\hline & & & Dimethyl phthalate & 0.8 & \\
\hline Meliaceae & Toona ciliata & Leaves, Stems & Diisobutyl phthalate & $\mathrm{N} / \mathrm{A}$ & [65] \\
\hline & & & 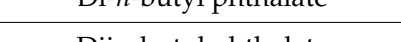 & $30-5$ & \\
\hline Orchidaceae & Cymbidium sinense & Flowers & Diisobutyl phthalate & 12.5 & [94] \\
\hline & & & Mono (2-ethylhexyl) phthalate & 26.5 & \\
\hline Olorс⿰丿е & Osmanthus fraorans & & Bis (2-methylpropyl) phthalate & 21.9 & {$[54]$} \\
\hline Oleaceae & Usmanthus fragrans & Flowers & Di- $n$-butyl phthalate & 15.1 & [54] \\
\hline & & & Diethyl phthalate & 2.1 & \\
\hline & & & Di- $n$-octyl phthalate & & \\
\hline Pontederiaceae & & Whole Plants & Diisooctyl phthalate & & \\
\hline Pontederiaceae & Eicnnornia crassipes & Whole Plants & Mono (2-ethylhexyl) phthalate & $\mathrm{N} / \mathrm{A}$ & [95] \\
\hline & & & Methyl dioctyl phthalate & & \\
\hline Polygonaceae & Polygonum amplexicaule & Roots & Diisobutyl phthalate & $\mathrm{N} / \mathrm{A}$ & [96] \\
\hline & & & Di-n-butyl phthalate & 7.0 & {$[44]$} \\
\hline Poaceae & Zea mays & Straws & 2-Methyl-pentyl-isobutyl & 6.4 & {$[44]$} \\
\hline & & & phthalate dibutyl & & \\
\hline
\end{tabular}


Table 2. Cont.

\begin{tabular}{|c|c|c|c|c|c|}
\hline Family & Identified from & Origin & Type of PAEs & $\begin{array}{l}\text { Relative Content } \\
\text { of PAEs (\%)* }\end{array}$ & References \\
\hline \multirow{4}{*}{ Rosaceae } & Malus prunifolia & Root Exudates & Phthalate derivates & 52.5 & [97] \\
\hline & Pyrus bretschneideri & Seeds & $\begin{array}{l}\text { Di- } n \text {-butyl phthalate } \\
\text { Diisobutyl phthalate }\end{array}$ & $\mathrm{N} / \mathrm{A}$ & {$[69]$} \\
\hline & Pyrus ussriensis & Fruits & Di(2-ethylhexyl) phthalate & 29.4 & {$[58]$} \\
\hline & Photinia parvifolia & Fruits & Di-n-octyl phthalate & 10.1 & {$[48]$} \\
\hline \multirow[t]{2}{*}{ Rubiaceae } & Paederia scandens & Whole Plants & $\begin{array}{l}\text { Di- } n \text {-butyl phthalate } \\
\text { Dimethyl phthalate } \\
\text { Diisobutyl phthalate } \\
\text { Di- } n \text {-octyl phthalate }\end{array}$ & $\begin{array}{l}5.0 \\
3.7 \\
3.2 \\
2.9\end{array}$ & [98] \\
\hline & Ixora amplexicaulis & Branches, Leaves & Di-n-butyl phthalate & 15.0 & {$[43]$} \\
\hline Rhamnaceae & Ziziphus mauritiana & Fruits & $\begin{array}{l}\text { Di(2-ethylhexyl) phthalate } \\
\text { Di- } n \text {-butyl phthalate }\end{array}$ & $\begin{array}{l}18.0 \\
12.3\end{array}$ & {$[59]$} \\
\hline \multirow{4}{*}{ Solanaceae } & Capsicum annuum & $\begin{array}{l}\text { Leaves and Root } \\
\text { Exudates }\end{array}$ & $\begin{array}{l}\text { Di- } n \text {-butyl phthalate } \\
\text { Butyl cyclohexane phthalate } \\
\text { Butyl isobutyl phthalate } \\
\text { Ditert butyl phthalate }\end{array}$ & $\begin{array}{l}41.5 \\
15.6 \\
13.1 \\
10.1\end{array}$ & [99] \\
\hline & Nicotiana tabacum & Root Exudates & $\begin{array}{l}\text { 3-hexyl isobutyl phthalate } \\
\text { Diisobutyl phthalate }\end{array}$ & $\begin{array}{l}4.8 \\
2.9\end{array}$ & {$[100]$} \\
\hline & Solanum lycopersicum & Root Exudates & $\begin{array}{l}\text { Di- } n \text {-butyl phthalate } \\
\text { Dimethyl phthalate } \\
\text { Diisooctyl phthalate } \\
\text { Diisobutyl phthalate }\end{array}$ & $\begin{array}{l}5.8 \\
2.1 \\
1.7 \\
0.4\end{array}$ & {$[101]$} \\
\hline & Solanum melongena & Root Exudates & $\begin{array}{l}\text { Di- } n \text {-butyl phthalate } \\
\text { Diisobutyl phthalate } \\
\text { Diisononyl phthalate }\end{array}$ & $\begin{array}{c}13.6 \\
1.9 \\
0.8\end{array}$ & {$[64]$} \\
\hline Saxifragaceae & Saxifraga stolonfera & Whole Plants & Butyloctyl phthalate & 5.5 & {$[102]$} \\
\hline Sargassaceae & Nizamuddinia zanardinii & Whole Plants & $\begin{array}{l}\text { Di- } n \text {-butyl phthalate } \\
\text { Diethyl phthalate }\end{array}$ & $\begin{array}{l}5.1 \\
0.7\end{array}$ & {$[103]$} \\
\hline Sapindaceae & Nephelium lappaceum & Peels & $\begin{array}{l}\text { Isobutyl octyl phthalate } \\
\text { Diisooctyl phthalate }\end{array}$ & $\begin{array}{c}16.5 \\
8.9\end{array}$ & {$[104]$} \\
\hline Salviniaceae & Salvinia natans & Whole Plants & $\begin{array}{l}\text { Mono (2-ethylhexyl) phthalate } \\
\text { Di- } n \text {-butyl phthalate }\end{array}$ & $\begin{array}{c}29.3 \\
1.0\end{array}$ & {$[105]$} \\
\hline Thymelaeaceae & Stellera chamaejasme & Root Exudates & $\begin{array}{l}\text { 2-Ethyl hexyl phthalate } \\
\text { Di- } n \text {-butyl phthalate } \\
\text { Diisobutyl phthalate }\end{array}$ & $\begin{array}{c}18.7 \\
4.6 \\
0.2\end{array}$ & {$[106]$} \\
\hline
\end{tabular}

* Relative Content of PAEs (\%) detected via GC/MS; ${ }^{* *}$ N/A: Not applicable.

\subsection{PAEs Identified and Purified from Microorganisms}

Phthalate compounds as bioactive natural products can be produced not only by plants, but also by bacteria and fungi (Table 3). Keire et al. (2001) [38] reported the first known example of diethyl phthalate produced by a bacterium, Helicobacter pylori, which represents a new class of immune-modulatory agent. Aboobaker et al. (2019) [107] isolated di-n-butyl phthalate as the major bioactive compound from the endophytic fungi, Pelargonium sidoides, which exhibits a significant inhibitory effect on Gram-positive bacteria (Staphylococcus aureus and Enterococcus faecalis) and Gram-negative bacteria (Escherichia coli and Pseudomonas aeruginosa). Rajamanikyam et al. (2017) [108] purified two PAEs, di(2-ethylhexyl) phthalate and di- $n$-butyl phthalate, from Brevibacterium mcbrellneri, both of which were isolated for the first time from the bacteria. Di- $n$-butyl phthalate was isolated from Streptomyces melanosporofaciens as an effective inhibitor of $\alpha$-glucosidase, which could provide useful reference information for the design of new effective inhibitors of glycosidase [109]. Furthermore, di(2-ethylhexyl) phthalate was isolated from Streptomyces bangladeshensis [110] and Penicillium olsonii [111]. Therefore, it is expected that PAEs can be characterized in various microorganisms, although their sources remain unclear. 
Table 3. PAEs purified from microorganisms.

\begin{tabular}{|c|c|c|c|c|}
\hline Category & Family & Species & Type of PAEs & References \\
\hline Bacteria & Brevibacteriaceae & Brevibacterium mcbrellneri & $\begin{array}{l}\text { Di(2-ethylhexyl) phthalate } \\
\text { Di- } n \text {-butyl phthalate }\end{array}$ & [108] \\
\hline Fungi & Davidiellaceae & Penicillium skrjabinii & Di-n-butyl phthalate & [107] \\
\hline Fungi & Davidiellaceae & Penicillium olsonii & Di(2-ethylhexyl) phthalate & [111] \\
\hline Bacteria & Helicobacteraceae & Helicobacter pylori & Diethyl phthalate & {$[38]$} \\
\hline Bacteria & Streptomycetaceae & Streptomyces melanosporofaciens & Di-n-butyl phthalate & [109] \\
\hline Bacteria & Streptomycetaceae & Streptomyces albidoflavus & Di-n-butyl phthalate & [112] \\
\hline Bacteria & Streptomycetaceae & Streptomyces bangladeshensis & Di(2-ethylhexyl) phthalate & [110] \\
\hline
\end{tabular}

\section{Biological Activities of PAEs}

\subsection{Allelopathic/Phytotoxic Activity}

Allelopathy refers to any direct or indirect harmful or beneficial effect exerted by one plant on another through the production of chemical compounds that are released into the environment. In some cases, allelopathy is suspected to contribute to the establishment of dominance of certain plant species, including some invasive alien species. Due to the phytotoxic property of allelochemicals, they are often considered valuable candidates for environmentally friendly bioherbicies $[113,114]$. Di-n-octyl phthalate isolated from Fimbristylis miliacea can remarkably inhibit the seed germination of tested weed species Ludwigia hysopifolia, Echinochloa colonum, Cyperus iria, and Paspalam digitatum [62]. Zhu et al. (2014) [72] isolated two allelochemicals, di(2-ethylhexyl) phthalate and di- $n$-butyl phthalate, from the root exudates of the invasive plant, Ageratina adenophora. In a bioassay, di- $n$-butyl phthalate was found to possess a significant inhibitory effect on seed germination and seedling growth of $A$. adenophora. Meanwhile, these two compounds significantly increased the superoxide dismutase (SOD) activity of $A$. adenophora's leaves and caused lipid peroxidation and cell membrane damage. Xuan et al. (2006) [115] identified the derivatives of phthalic acids from root exudates of Echinochloa crusgalli and found that diethyl phthalate strongly affects the seedling growth of alfalfa, Indian jointvetch, lettuce, monochorea, and sesame. Huang et al. (2017) [116] analyzed the extracts of aerial parts plants, root exudates, and plant rhizosphere soil of Chrysanthemum indicum to determine the effect of the allelochemical diethyl phthalate, and the results show that it has a noticeable impact on promoting the fresh weight of lettuce, as well as the root growth of lettuce and rape. Shanab et al. (2010) [95] extracted four phthalate derivatives from Eichhornia crassipes, including di- $n$-octyl phthalate, mono (2-ethylhexyl) phthalate, methyl dioctyl phthalate, and diisooctyl phthalateis, which possess strong inhibitory effects on Chlorella vulgaris.

Physiological studies have indicated that PAEs can influence enzyme activity, which might be at least one of their phytotoxicity mechanisms. Deng et al. (2017) [117] revealed that as the concentration of PAEs secreted by tobacco roots increased, the rate of production of superoxide anion radicals, the concentration of malondialdehyde, and the activity of peroxidase and SOD in tobacco root increased significantly. A series of changes could reduce the root system's antioxidant properties and cause oxidative damage to the apical cell membrane system, thereby affecting root absorption and ultimately showing autotoxicity. Dong et al. (2016) [67] extracted diisobutyl phthalate and di- $n$-butyl phthalate from the ethyl acetate extract of $C$. fracta, both of which show a strong inhibitory effect on the growth of Heterosigma akashiwo and Gymnodinium breve, which may be related to the production of reactive oxygen species (ROS) induced by diisobutyl phthalate and di- $n$-butyl phthalate in algal cells. Excessive ROS inhibits the activities of catalase and SOD, leading to lipid oxidation and the destruction of algae cell membranes.

\subsection{Antimicrobial Activity}

Natural products, including secondary metabolites produced by plants and microorganisms, have long been studied for their antimicrobial activity in the search for eco-friendly substitutes for synthesized chemicals [118]. Di(2-ethylhexyl) phthalate and di- $n$-butyl 
phthalate isolated from B. mcbrellneri show broad-spectrum antibacterial activity [108]. Di(2-ethylhexyl) phthalate can inhibit the growth of gram-positive (S. epidermidis, MIC of $9.37 \mu \mathrm{g} / \mathrm{mL}$; S. aureus, MIC of $18.75 \mu \mathrm{g} / \mathrm{mL}$ ) and gram-negative bacteria (E. coli, MIC of $37.5 \mu \mathrm{g} / \mathrm{mL}$; P. aeruginosa and Klebsiella pneumoniae, MIC at $75 \mu \mathrm{g} / \mathrm{mL}$ for both). Di$n$-butyl phthalate also inhibits the growth of gram-positive (Bacillus subtilis and S. epidermidis, MIC at $18.75 \mu \mathrm{g} / \mathrm{mL}$ for both) as well as gram-negative bacteria (E. coli and P. aeroginosa, MIC at $37.5 \mu \mathrm{g} / \mathrm{mL}$ for both). Di(2-ethylhexyl) phthalate isolated from the flowers of Calotropis gigantean exerts antimicrobial activity against $B$. subtilis with a MIC of $32 \mu \mathrm{g} / \mathrm{mL}$ [119]. There are also reports on the antimicrobial activity of di- $n$-butyl phthalate isolated from Streptomyces albidoflavus showing a MIC for E. coli of $53 \mu \mathrm{g} / \mathrm{mL}$, with B. subtilis at $84 \mu \mathrm{g} / \mathrm{mL}$ [112]. Four phthalate derivatives isolated from E. crassipes also exert significant antibacterial activity against gram-positive bacteria (B. subtilis and Streptococcus faecalis) and gram-negative bacteria E. coli, and antifungal activity against Candida albicans [95]. In another study, El-Mehalawy et al. (2008) [120] found that di(2-ethylhexyl) phthalate could be produced by certain bacteria, including Tsukamurella inchonensis, Corynebacterium nitrilophilus, and Cellulosimicrbium cellulans, and di(2-ethylhexyl) phthalate has the function to inhibit fungal spore germination, cell membrane growth, and the production of total lipids and total protein. Li et al. (2021) [121] isolated di- $n$-butyl phthalate from a new marine Streptomyces sp. and found this compound significantly inhibited spore germination and mycelial growth of Colletotrichum fragariae. In addition to this, an obvious decrease was detected in sugar and protein contents of $C$. fragariae mycelia. Other studies have shown similar results. For instance, di- $n$-butyl phthalate was reported to inhibit spore germination and mycelium growth of Colletotrichum gloeosporioides, Colletotrichum musae, and Gaeumannomyces graminis [122-124].

Janu and Jayanthy (2014) found that diethyl phthalate derived from the fungus Aspergillus sp. increased the superoxide production and exerted ROS generated oxidative stress in the cytoplasm of bacterial cells, which eventually led to cell death [125]. In addition, diethyl phthalate with antimicrobial properties was reported for its ability to interfere with quorum sensing mediated virulence factors and biofilm formation in Pseudomonas aeruginosa $[126,127]$. Another study demonstrated that dimethyl phthalate (concentration ranged from 20 to $40 \mathrm{mg} / \mathrm{L}$ ) greatly inhibited the growth and glucose utilization of Pseudomonas fluorescens, meanwhile the surface hydrophobicity and membrane permeability of $P$. fluorescens were also increased. Dimethyl phthalate could lead to deformation of the cell membrane and misopening of membrane channels. Additionally, RNA-Seq and RT-qPCR results revealed that the expression of some genes in P. fluorescens were altered, including the genes involved in energy metabolism, ATP-binding cassette transporting, and two-component systems by dimethyl phthalate [128].

\subsection{Insecticidal Activity}

In addition to their phytotoxic and antimicrobial activity, PAEs were also found to be insecticidal; attributed to inhibition of acetylcholinesterase enzyme activity, they possess significant mosquito larvicidal activity. Therefore, some phthalates, such as synthetic diethyl phthalate and dimethyl phthalate, have been used as active ingredients in insect repellents [129,130]. Previously, Adsul et al. (2012) [79] isolated di- $n$-butyl phthalate from the leaf extract of Ipomoea carnea via column chromatography, and this compound ws found to be lethal to the fourth instar larvae of Aedes aegypti and Culex quinquefasciatus, with the lethal concentrations of $\mathrm{LC}_{50}$ being 81.43 and $109.64 \mathrm{ppm}$, respectively. Di- $n$-butyl phthalate and di(2-ethylhexyl) phthalate were isolated from the bacterium B. mcbrellneri; because of their significant acetylcholinesterase inhibitory activity, they are also active against the fourth instar of $A$. aegypti after $24 \mathrm{~h}$ of exposure [108]. On the other hand, various PAEs were also constantly reported as possessing other biological activities, such as anti-inflammatory, antiviral, anti-tumor, antidiabetic activity, etc., indicating their valuable potential to be explored further in capacities other than plasticizers [17] (Figure 3). 


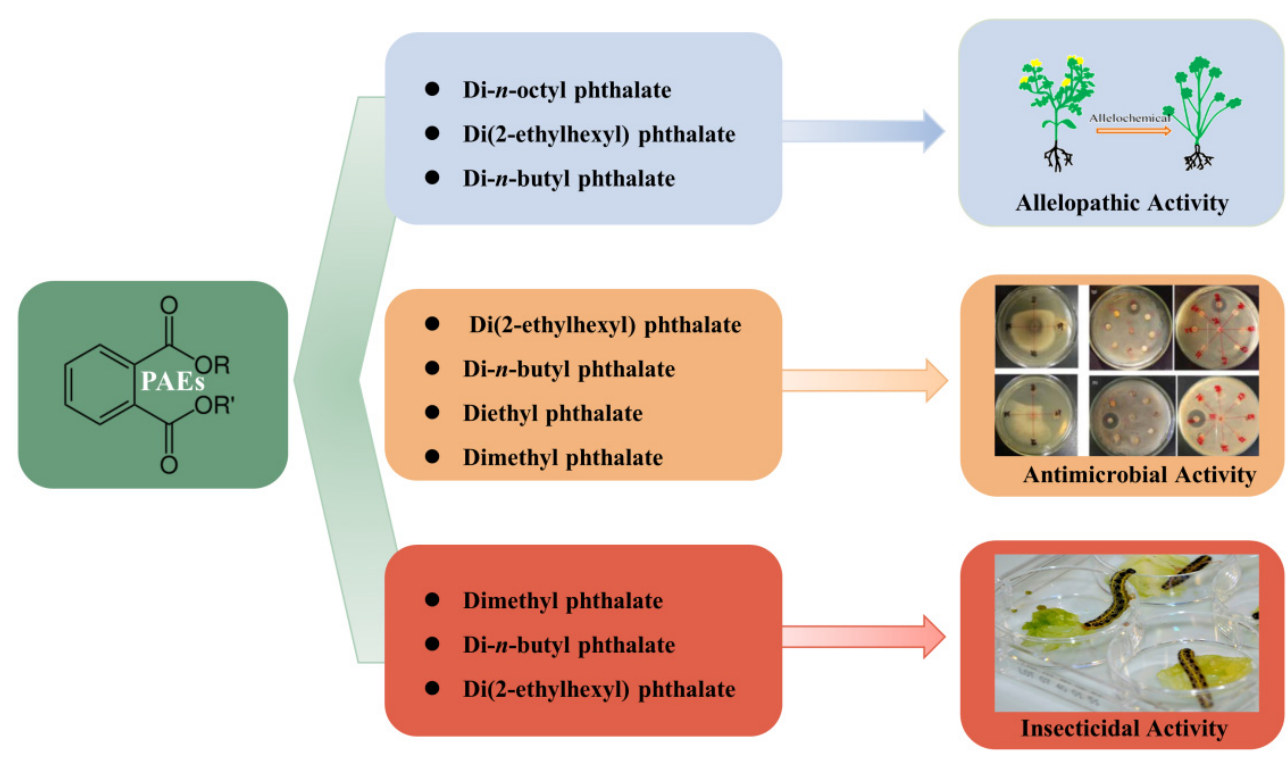

Figure 3. Biological activities of PAEs in living organisms.

\section{Conclusions and Perspectives}

PAEs have attracted attention due to their ubiquitous presence in environmental media. Many phthalates have been reported in nature, such as sediments, natural water, soil, aquatic organisms, etc. $[17,20,30,34]$. On the other hand, as phthalates exist in many laboratory products, such as instruments, reagents, solvents, and consumables, source detection also poses analytical challenges [131-133]. PAEs in the environment are mainly derived from chemical syntheses that are applied in building materials, care products, medical equipment, and children's toys, etc., which are convenient for human production and life $[19,27,132]$. However, the published literature also indicated that PAEs can be synthesized naturally, and they might serve as biologically active substances to enhance competitiveness. The isotope labeling approach has demonstrated that PAEs can be biosynthesized by several algae, possibly via the shikimic acid pathway [134-136]. Ecologically, PAEs with allelopathic activity might facilitate the establishment of the dominance of plants or algae that are capable of producing them. In addition, phthalate esters' insecticidal activity protects plants from being consumed by insects $[17,129,137]$, not to mention the fact that the antimicrobial activity of PAEs may reduce the damage caused by pathogenic fungi and bacteria $[17,107,108,118,119]$.

Studies have shown that some algae can also synthesize phthalates to defend against biotic and abiotic factors. Babu and Wu (2010) [138] highlighted that some freshwater algae and cyanobacteria species are capable of producing di- $n$-butyl phthalate and mono (2-ethylhexyl) phthalate. These phthalates may be released into the environment under pressure, affecting the aquatic ecosystem. The above conclusion is in good agreement with Chen's results, who reported on the de novo synthesis of di(2-ethylhexyl) phthalate and di- $n$-butyl phthalate in a marine alga. In algal cells, biosynthesized PAEs are presumably stored in cell membranes to maintain the flexibility of algal cells [134]. These findings suggest that the production of phthalates may be a common phenomenon on both land and in the sea. Meanwhile, some PAEs identified in the root exudates of various crops could effectively reduce soil-borne diseases, improve soil properties, and promote plant growth $[64,101,139]$. Nevertheless, the biosynthetic pathways of these secondary metabolites are highly complex and are the result of the combined actions of biotic and abiotic stressors, which are worthy of in-depth study by phytochemical researchers $[134,138,140]$.

In conclusion, PAEs are widespread around us, not only from synthetic materials but also from living organisms, such as microbes, algae, plants, etc. Chemically synthesized PAEs have been widely applied in industry to improve the quality of various products. In contrast, naturally synthesized PAEs can potentially serve as allelochemicals, antibiotics, 
or insecticides to increase the adaptability of donor species. It is challenging to quantify the PAEs around us, and the amount of PAEs in the environment will continue to rise. We know that PAEs can be synthesized naturally, which implies that certain microorganisms are capable of degrading them, and their potential is worthy of further study to reduce PAEs' contamination of the environment.

Author Contributions: Conceptualization, X.Z. and L.H.; writing-original draft preparation, L.H., X.Z., S.Z., Z.C. and K.S.; writing-review and editing, H.S. and C.Z.; funding acquisition, H.S. and C.Z. All authors have read and agreed to the published version of the manuscript.

Funding: This study was financially supported by the Taishan Scholars Program of Shandong, China (Grant No. ts201712071); the Open Fund of Shandong Provincial Key Laboratory of Water and Soil Conservation and Environmental Protection, China, Grant No. STKF201935; and the National Natural Science Foundation of China (31770586).

Institutional Review Board Statement: Not applicable.

Informed Consent Statement: Not applicable.

Data Availability Statement: Not applicable.

Conflicts of Interest: The authors declare no conflict of interest.

\section{References}

1. Jeddi, M.Z.; Janani, L.; Memari, A.H.; Akhondzadeh, S.; Yunesian, M. The role of phthalate esters in autism development: A systematic review. Environ. Res. 2016, 151, 493-504. [CrossRef]

2. He, Y.; Wang, Q.; He, W.; Xu, F. The occurrence, composition and partitioning of phthalate esters (PAEs) in the water-suspended particulate matter (SPM) system of Lake Chaohu, China. Sci. Total Environ. 2019, 661, 285-293. [CrossRef]

3. Chi, Z.; Zhao, J.; Li, W.; Araghi, A.; Tan, S. In vitro assessment of phthalate acid esters-trypsin complex formation. Chemosphere 2017, 185, 29-35. [CrossRef] [PubMed]

4. Fan, Y.; Chen, H.; Liu, H.; Wang, F.; Ma, S.; Latipa, A.; Wang, S.; Wang, C. Analysis of phthalate esters in dairy products-a brief review. Anal. Methods 2017, 9, 370-380. [CrossRef]

5. Sardon, H.; Dove, A.P. Plastics recycling with a difference. Science 2018, 360, 380-381. [CrossRef] [PubMed]

6. Wang, W.; Themelis, N.J.; Sun, K.; Bourtsalas, A.C.; Huang, Q.; Zhang, Y.; Wu, Z. Current influence of China's ban on plastic waste imports. Waste Dispos. Sustain. Energy 2019, 1, 67-78. [CrossRef]

7. Gao, D.W.; Wen, Z.D. Phthalate esters in the environment: A critical review of their occurrence, biodegradation, and removal during wastewater treatment processes. Sci. Total Environ. 2016, 541, 986-1001. [CrossRef] [PubMed]

8. Gani, K.M.; Tyagi, V.K.; Kazmi, A.A. Occurrence of phthalates in aquatic environment and their removal during wastewater treatment processes: A review. Environ. Sci. Pollut. Res. 2017, 24, 17267-17284. [CrossRef]

9. Miodovnik, A.; Edwards, A.; Bellinger, D.C.; Hauser, R. Developmental neurotoxicity of ortho-phthalate diesters: Review of human and experimental evidence. Neurotoxicology 2014, 41, 112-122. [CrossRef]

10. Howdeshell, K.L.; Wilson, V.S.; Furr, J.; Lambright, C.R.; Rider, C.V.; Blystone, C.R.; Hotchkiss, A.K.; Gray, L.E., Jr. A mixture of five phthalate esters inhibits fetal testicular testosterone production in the sprague-dawley rat in a cumulative, dose-additive manner. Toxicol. Sci. 2008, 105, 153-165. [CrossRef]

11. Heudorf, U.; Mersch-Sundermann, V.; Angerer, J. Phthalates: Toxicology and exposure. Int. J. Hyg. Environ. Health 2007, 210, 623-634. [CrossRef] [PubMed]

12. Wang, Y.; Zhang, G.; Wang, L. Potential Toxicity of Phthalic Acid Esters Plasticizer: Interaction of Dimethyl Phthalate with Trypsin in Vitro. J. Agric. Food Chem. 2015, 63, 75-84. [CrossRef] [PubMed]

13. Xu, H.; Shao, X.; Zhang, Z.; Zou, Y.; Chen, Y.; Han, S.; Wang, S.; Wu, X.; Yang, L.; Chen, Z. Effects of di-n-butyl phthalate and diethyl phthalate on acetylcholinesterase activity and neurotoxicity related gene expression in embryonic zebrafish. Bull. Environ. Contam. Toxicol. 2013, 91, 635-639. [CrossRef] [PubMed]

14. Xu, H.; Shao, X.; Zhang, Z.; Zou, Y.; Wu, X.; Yang, L. Oxidative stress and immune related gene expression following exposure to di-n-butyl phthalate and diethyl phthalate in zebrafish embryos. Ecotoxicol. Environ. Saf. 2013, 93, 39-44. [CrossRef] [PubMed]

15. Zhao, X.; Gao, Y.; Qi, M. Toxicity of phthalate esters exposure to carp (Cyprinus carpio) and antioxidant response by biomarker. Ecotoxicology 2014, 23, 626-632. [CrossRef]

16. Schmid, H.; Karrer, P. The Water-Soluble Constituents of Papaver Somniferum L. Helv. Chim. Acta 1945, 28, 722-740. [CrossRef]

17. Roy, R.N. Bioactive natural derivatives of phthalate ester. Crit. Rev. Biotechnol. 2020, 40, 913-929. [CrossRef]

18. Mathur, S.P. Phthalate esters in the environment: Pollutants or natural products? J. Environ. Qual. 1974, 3, 189-197. [CrossRef]

19. Gomez-Hens, A.; Aguilar-Caballos, M.P. Social and economic interest in the control of phthalic acid esters. TrAC Trends Anal. Chem. 2003, 22, 847-857. [CrossRef] 
20. Net, S.; Sempere, R.; Delmont, A.; Paluselli, A.; Ouddane, B. Occurrence, Fate, Behavior and Ecotoxicological State of Phthalates in Different Environmental Matrices. Environ. Sci. Technol. 2015, 49, 4019-4035. [CrossRef]

21. Autian, J. Toxicity and health threats of phthalate esters: Review of the literature. Environ. Health Perspect. 1973, 4, 3-26. [CrossRef]

22. Das, M.T.; Kumar, S.S.; Ghosh, P.; Shah, G.; Malyan, S.K.; Bajar, S.; Thakur, I.S.; Singh, L. Remediation strategies for mitigation of phthalate pollution: Challenges and future perspectives. J. Hazard. Mater. 2020, 124496. [CrossRef]

23. Hu, A.P.; Liu, Y.L.; Shi, L.K. Widespread occurrence of phthalic acid esters in raw oilseeds in China used for edible vegetable oil production. Food Addit. Contam. Part A 2016, 33, 1421-1427. [CrossRef]

24. Giuliani, A.; Zuccarini, M.; Cichelli, A.; Khan, H.; Reale, M. Critical review on the presence of phthalates in food and evidence of their biological impact. Int. J. Environ. Res. Public Health. 2020, 17, 5655. [CrossRef] [PubMed]

25. Vieira, M.G.A.; da Silva, M.A.; dos Santos, L.O.; Beppu, M.M. Natural-based plasticizers and biopolymer films: A review. Eur. Polym. J. 2011, 47, 254-263. [CrossRef]

26. Alnaimat, A.S.; Barciela-Alonso, M.C.; Bermejo-Barrera, P. Development of a sensitive method for the analysis of four phthalates in tea samples: Tea bag contribution to the total amount in tea infusion. Food Addit. Contam. Part A 2020, 37, 1719-1729. [CrossRef]

27. Martínez, M.; Rovira, J.; Sharma, R.P.; Nadal, M.; Schuhmacher, M.; Kumar, V. Comparing dietary and non-dietary source contribution of BPA and DEHP to prenatal exposure: A Catalonia (Spain) case study. Environ. Res. 2018, 166, 25-34. [CrossRef] [PubMed]

28. Chen, H.; Mao, W.; Shen, Y.; Feng, W.; Mao, G.; Zhao, T.; Yang, L.; Yang, L.; Meng, C.; Li, Y.; et al. Distribution, source, and environmental risk assessment of phthalate esters (PAEs) in water, suspended particulate matter, and sediment of a typical Yangtze River Delta City, China. Environ. Sci. Pollut. Res. 2019, 26, 24609-24619. [CrossRef]

29. Sha, Y.; Xia, X.; Yang, Z.; Huang, G.H. Distribution of PAEs in the middle and lower reaches of the Yellow River, China. Environ. Monit. Assess. 2007, 124, 277-287. [CrossRef] [PubMed]

30. Liu, Y.; Ji, C.; Yu, Y.; Liu, H.; Shen, Y. Distribution characteristics and health risk assessment of PAEs in urban soils of Changji City, Xinjiang, China. Chin. J. Nat. 2020, 22, 67-74. [CrossRef]

31. Wang, H.; Liang, H.; Gao, D.W. Occurrence and risk assessment of phthalate esters (PAEs) in agricultural soils of the Sanjiang Plain, northeast China. Environ. Sci. Pollut. Res. 2017, 24, 19723-19732. [CrossRef]

32. He, Y.; Wang, Q.; He, W.; Xu, F. Phthalate esters (PAEs) in atmospheric particles around a large shallow natural lake (Lake Chaohu, China). Sci. Total Environ. 2019, 687, 297-308. [CrossRef]

33. Wang, P.; Wang, S.L.; Fan, C.Q. Atmospheric distribution of particulate- and gas-phase phthalic esters (PAEs) in a Metropolitan City, Nanjing, East China. Chemosphere 2008, 72, 1567-1572. [CrossRef]

34. Zhao, X.; Jin, H.; Ji, Z.; Li, D.; Kaw, H.Y.; Chen, J.; Xie, Z.; Zhang, T. PAES and PAHs in the surface sediments of the East China Sea: Occurrence, distribution and influence factors. Sci. Total Environ. 2020, 703, 134763. [CrossRef] [PubMed]

35. Arfaeinia, H.; Fazlzadeh, M.; Taghizadeh, F.; Saeedi, R.; Spitz, J.; Dobaradaran, S. Phthalate acid esters (PAEs) accumulation in coastal sediments from regions with different land use configuration along the Persian Gulf. Ecotoxicol. Environ. Saf. 2019, 169, 496-506. [CrossRef]

36. Bu, S.B.; Wang, Y.L.; Wang, H.Y.; Wang, F.; Tan, Y.F. Analysis of global commonly-used phthalates and non-dietary exposure assessment in indoor environment. Build. Environ. 2020, 177, 106853. [CrossRef]

37. Hu, H.; Fang, S.; Zhao, M.; Jin, H. Occurrence of phthalic acid esters in sediment samples from East China Sea. Sci. Total Environ. 2020, 722, 137997. [CrossRef]

38. Keire, D.A.; Anton, P.; Faull, K.F.; Ruth, E.; Walsh, J.H.; Chew, P.; Quisimoro, D.; Territo, M.; Reeve, J.R. Diethyl phthalate, a chemotactic factor secreted by Helicobacter pylori. J. Biol. Chem. 2001, 276, 48847-48853. [CrossRef] [PubMed]

39. Li, F.M.; Wu, M.; Yao, Y.; Zheng, X.; Zhao, J.; Wang, Z.Y.; Xing, B.S. Inhibitory effects and oxidative target site of dibutyl phthalate on Karenia brevis. Chemosphere 2015, 132, 32-39. [CrossRef] [PubMed]

40. Daiem, M.M.A.; Rivera-Utrilla, J.; Ocampo-Perez, R.; Mendez-Diaz, J.D.; Sanchez-Polo, M. Environmental impact of phthalic acid esters and their removal from water and sediments by different technologies-A review. J. Environ. Manag. 2012, 109, 164-178. [CrossRef]

41. Martinez-Razo, L.D.; Martinez-Ibarra, A.; Vazquez-Martinez, E.R.; Cerbon, M. The impact of Di-(2-ethylhexyl) Phthalate and Mono(2-ethylhexyl) Phthalate in placental development, function, and pathophysiology. Environ. Int. 2021, 146, 106228. [CrossRef]

42. Zhang, X.P.; Ning, T.Y.; Zhang, J.Y.; Yang, Y.; Sun, T.; Han, H.F.; Li, Z.J. Allelopathy of Decomposed Exudates from Broccoli Stalk on Seedlings Growth of Different Vegetables. J. Shandong Agric. Univ. Nat. Sci. Ed. 2016, 47, 481-486.

43. Chen, L.; Yue, Y.; Jiang, C.; Jiang, J.; Chen, Z.; Chen, Y. GC-MS analysis of liposoluble constituents from Iroxa amplexicaulis. China J. Tradit. Chin. Med. Pharm. 2011, 26, 3003-3005.

44. Qi, Y.Z.; Zhen, W.C.; Li, H.Y. Allelopathy of decomposed maize straw products on three soil-born diseases of wheat and the analysis by GC-MS. J. Integr. Agric. 2015, 14, 88-97. [CrossRef]

45. Li, Y.B.; Zhang, Q.; Wan, C.X.; Gong, M.F.; Zhang, L.L. Allelopathy and chemical constituents of decomposing products from cotton stalk. Cotton Sci. 2009, 21, 497-502.

46. Tian, Q.; Liang, Z.; Chen, Y.; Wang, J.; Deng, S. Analysis of Petroleum Ether Extract from Jatropha curcas Leaves by GC-MS. Lishizhen Med. Mater. Med. Res. 2011, 22, 2117-2118. 
47. Zhao, X.; Lou, Y.; Su, F.; Zhou, C.; Zhang, Y. Study on the components with hypotensive activities from petroleum ether part of compound Prunella vulgaris L. Chin. J. Hosp. Pharm. 2014, 34, 1-4. [CrossRef]

48. Cheng, Z.; Yi, X.; Tan, D.; Lou, X.; Yang, D. GC-MS analysis of the low-and-middle polarity components from the fruit of Photinia parvifolia. Guihaia 2013, 33, 568-570.

49. Rubiolo, P.; Sgorbini, B.; Liberto, E.; Cordero, C.; Bicchi, C. Essential oils and volatiles: Sample preparation and analysis. A review. Flavour Fragr. J. 2010, 25, 282-290. [CrossRef]

50. Burt, S. Essential oils: Their antibacterial properties and potential applications in foods-A review. Int. J. Food Microbiol. 2004, 94, 223-253. [CrossRef]

51. Bakkali, F.; Averbeck, S.; Averbeck, D.; Idaomar, M. Biological effects of essential oils-A review. Food Chem. Toxicol. 2008, 46, 446-475. [CrossRef]

52. Wu, J.; Chen, T.; Qin, L. Chemical Constituents of the Essential Oil from Radix Pseudostellariae (I). Nat. Prod. Res. Dev. 2008, 20, 458-460, 487. [CrossRef]

53. Kirillov, V.; Stikhareva, T.; Atazhanova, G.; Serafimovich, M.; Mukanov, B.; Adekenov, S.; Mukasheva, F.; Yrymgali, M. Chemical Composition of the Essential Oil of the Boreal Relict of Pyrola rotundifolia L. from Northern Kazakhstan. J. Oleo Sci. 2015, 64, 1065-1073. [CrossRef]

54. Xu, J.M.; Lu, J.S. A study on chemical composition of the essential oils from Osmanthus fragrant flowers. Anal. Lab. 2007, $26,37-41$.

55. Gan, Y.; Liu, G. Study on the essential chemical component of giant taro, Alocasia macrorrhiza (L.) Schott. Guangdong Agric. Sci. 2012, 39, 38-39. [CrossRef]

56. Li, D.; Wang, Z.; Liang, Z.; Yang, X.; Xu, J. Comparative evaluation of the chemical composition of essential oil from twig, leaf and root of Clerodendrum inerme (L.) Gaertn. Adv. Mater. Res. 2012, 343, 22-27. [CrossRef]

57. Luo, X.; Yang, Z.R. Studies on chemical components and antibacterial activity of essential oil from Cirsium japonicum DC. J. Sichuan Univ. Nat. Sci. Ed. 2009, 46, 1531-1536.

58. Xin, Y.; Liu, C.; Hou, D.; Li, T. Analysis of the Chemical Constituents of Essential Oil of Flesh from Nanguoli Pear. Food Sci. 2004, 25, 223-225.

59. Deng, G.; Li, X.; Lin, Y.; Han, X.; Zhang, X. Study of the Volatile Components of the Fruit of Ziziphus mauritiana Lam.by GC-MS. Fine Chem. 2004, 21, 318-320.

60. Bertin, C.; Yang, X.H.; Weston, L.A. The role of root exudates and allelochemicals in the rhizosphere. Plant Soil 2003, 256, 67-83. [CrossRef]

61. Canarini, A.; Kaiser, C.; Merchant, A.; Richter, A.; Wanek, W. Root Exudation of Primary Metabolites: Mechanisms and Their Roles in Plant Responses to Environmental Stimuli. Front. Plant. Sci. 2019, 10, 157. [CrossRef]

62. Ismail, B.S.; Siddique, A.B. Identification of allelochemicals from Fimbristylis miliacea and their allelopathic potential against weed species. Allelopath. J. 2012, 30, 311-318.

63. Cheng, Z.; Xu, P. GC-MS identification of chemicals in lily root exudates. J. Northwest A F. Univ. Nat. Sci. Ed. 2012, 40, 202-208. [CrossRef]

64. Zhou, B.L.; Yin, Y.L.; Zhang, F.L.; Ye, X.L. Allelopathic effects of root exudates of grafted eggplants on Verticillium dahliae and their constituents' identification. Allelopath. J. 2010, 25, 393-402.

65. Liu, Y.B.; Cheng, X.R.; Qin, J.J.; Yan, S.K.; Jin, H.Z.; Zhang, W.D. Chemical constituents of Toona ciliata var. pubescens. Chin. J. Nat. Med. 2011, 9, 115-119. [CrossRef]

66. Shi, D.; Han, L.; Sun, J.; Wang, Y.; Yang, Y.; Shi, J.; Fan, X. Chemical constituents from marine alga Chaetomorpha basiretorsa. China J. Chin. Mater. Med. 2005, 30, 347-350.

67. Dong, S.J.; Bi, X.D.; Wang, N.; Song, L.; Dai, W.; Zhang, S.L. Algicidal activities of Cladophora fracta on red tide-forming microalgae Heterosigma akashiwo and Gymnodinium breve. Allelopath. J. 2016, 37, 231-240.

68. Pan, Z.; Wu, Y.; Ning, D.; Wei, Y. Chemical constituents from the root of Croton lachynocarpus. Guihaia 2014, 34, 148-150, 255.

69. Li, X.; Zhang, J.; Gao, W.; Wang, H. Study on chemical composition, anti-inflammatory and anti-microbial activities of extracts from Chinese pear fruit (Pyrus bretschneideri Rehd.). Food Chem. Toxicol. 2012, 50, 3673-3679. [CrossRef]

70. Huang, L.S.; Zhu, F.; Huang, M.Z. GC/MS Analysis of the Chemical Constituents of the Essential Oil from the Fruits of Avicennia Marina. Fine Chem. 2009, 26, 255-257.

71. Olufunke, M.D. Essential Oils from Aerial, Seed and Root of Nigerian Asystasia gangetica (L). J. Essent. Oil-Bear. Plants. 2011, 14, 582-589. [CrossRef]

72. Zhu, X.Z.; Guo, J.; Shao, H.; Yang, G.Q. Effects of allelochemicals from Ageratina adenophora (Spreng.) on its own autotoxicity. Allelopath. J. 2014, 34, 253-264.

73. Jiang, P.; Zheng, D.F.; Tian, W.; Du, N.N.; Zhang, X.; Zhang, A.L. Identification of allelochemicals in Chrysanthemum indicum L. and their fungicidal potential against Sclerotium rolfsii Sacc. and Atractylodes macrocephala Koidz. Allelopath. J. 2016, 37, 137-145.

74. Ma, J.P.; Guo, Z.B.; Jin, L.; Li, Y.D. Phytochemical progress made in investigations of Angelica sinensis (Oliv.) Diels. Chin. J. Nat. Med. 2015, 13, 241-249. [CrossRef]

75. Li, C.; Chen, M.; Ma, F.; Gai, Z.; Guo, J.; Wang, Y. Study on allelopathy of sugar beet root exudates on soybean. J. Northeast Agric. Univ. 2016, 47, 21-30. [CrossRef] 
76. Zhao, C.; Zhang, M.; Xiang, C.; Li, B. Analysis on chemical constituents in essential oil from Campanula colorata. J. Plant Resour. Environ. 2014, 23, 99-101.

77. Si, H.Q.; Shen, Q.; Pang, X.L. Supercritical Fluid Carbon Dioxide Extraction and GC-MS Analysis of Essential Oil from Chimonanthus praecox Flowers. Food Sci. 2010, 31, 134-137.

78. Wang, Y.; Tang, Z.; Cao, M.; Li, Y.; Zhang, T.; Du, H. Allelopathic effects of Hylotelephium erythrostictum flowers on three weed species and identification of potential allelochemicals. Acta Pratacult. Sin. 2020, 29, 175-182.

79. Adsul, V.B.; Khatiwora, E.; Torane, R.C.; Deshpande, N.R. Isolation and characterization of dibutyl phthalate from leaves of Ipomoea carnea. Chem. Nat. Compd. 2012, 48, 712-713. [CrossRef]

80. Tian, P.; Fu, X.; Zhuang, P.; Bai, J.; Chen, F. Analysis on Volatile Oil from Rhododendron calophytum Franch by GC-MS. Chin. J. Appl. Environ. Biol. 2010, 16, 734-737.

81. Chen, L.X.; Liu, S.H.; Chen, X.; Peng, L.X. Chemical Composition and Antibacterial Activity of the Essential Oils from Dalbergia odorifera T.Chen. Chin. J. Trop. Crops. 2011, 32, 1165-1167.

82. Zhao, R.; Cai, L. The Determination of Root Exudates of Medicago sativa and the Study on Allelopathic Effect of Typical Exudation 3,5-di-tert-Butyl-4-hydroxybenzaldehyde. Chin. Agri. Sci. Bull. 2013, 29, 34-41.

83. Lu, H.; Xie, H.; Yang, Y.; Wei, X. Chemical Constituents from the Macroalga Gracilaria lemaneiformis. J. Trop. Subtrop. Bot. 2011, 19, 166-170.

84. Li, J.L.; Liu, J.H.; Gao, Y.Q.; Huo, X.; Wang, D.P.; Sheng, S.C. Study on Chemical Constituents of Volatile Oil from Lysionotus pauciflorus. Chin. Pharm. 2011, 22, 2560-2562.

85. Shafaghat, A. Omega-3 content, antimicrobial and antioxidant activities of hexanic extract from seed and leaf of Hypericum scabrum from northwestern Iran. Afr. J. Microbiol. Res. 2012, 6, 904-908. [CrossRef]

86. Xu, N.; Wang, C.; Wei, M.; Shi, W.; Wang, X. Allelopathy of Welsh Onion Root Exudates on Cucumber Seed Germination and Fusarium oxysporum f. sp. cucumerinum and the GC-MS Analysis. Acta Hortic. Sin. 2012, 39, 1511-1520. [CrossRef]

87. Liu, Z.; Liu, Z.; Tian, Q. Analysis of Chemical Components of Volatile Oil from Paris polyphylla and Their Antibacterial Activities. J. Chin. Med. Mater. 2014, 37, 612-616. [CrossRef]

88. Efremov, A.A.; Zykova, I.D.; Gorbachev, A.E. Composition of the essential oil from the lemon balm growing in the neighborhood of Krasnoyarsk as indicated by gas chromatography-mass spectrometry data. Russ. J. Bioorg. Chem. 2016, 42, 726-729. [CrossRef]

89. Naidoo, Y.; Sadashiva, C.T.; Kasim, N.; Nicholas, A.; Naidoo, G. Chemical Composition and Antimicrobial Activity of the Essential Oil of Ocimum obovatum E. Mey. Ex Benth. (Lamiaceae). J. Essent. Oil-Bear. Plants. 2014, 17, 142-147. [CrossRef]

90. Tian, G.H.; Liu, C.F.; Wei, C.; Lai, P.H. Study on component analysis and antimicrobial activity of the essential oil of the flower of Phlomis umbrosa. Chin. J. Pharm. Anal. 2009, 29, 390-394.

91. Yu, Z.X.; Wang, G.L.; Dai, Z.; Bianba, C.R.; Lin, R. Studies on Chemical Constituents ef Phlomis medicinalis II. Chin. Pharm. J. 2007, 42, 1295-1298.

92. Cao, Y.; Zhang, D.; Yang, L.; Xue, P.; Yang, L.; Zhang, K.; Liang, C. Analysis of the Volatile Compounds in Scutellaria barbata Using HS-SPME-GC-MS. Chin. J. Exp. Tradit. Med. Formulae 2015, 21, 40-43. [CrossRef]

93. Liu, T.; Jin, J.; Jin, J.; Peng, Z.; Luo, F. Volatile Constituents from Myricarubra cv. DingAo Orient Pearl and Its Antitumor Activity. Nat. Prod. Res. Dev. 2014, 26, 1839-1842. [CrossRef]

94. Li, J.; Wang, Z.; Zhang, J.; Zhu, G. Composition Analysis on Flower Essential Oils of Cymbidium sinense 'Qi Hei'. Chin. J. Trop. Crops. 2016, 37, 86-91.

95. Shanab, S.M.; Shalaby, E.A.; Lightfoot, D.A.; El-Shemy, H.A. Allelopathic effects of water hyacinth [Eichhornia crassipes]. PLoS ONE 2010, 5, e13200. [CrossRef] [PubMed]

96. Xiang, M.; Hu, Y.; Yan, Y. Study on Chemical Composition of Ethylacetate Fraction from Polygonum amplexicaule var.sinense. J. Chin. Med. Mater. 2012, 35, 1610-1614. [CrossRef]

97. Bai, R.; Zhao, X.; Ma, F.; Li, C. Identification and bioassay of allelopathic substances from the root exudates of Malus prunifolia. Allelopath. J. 2009, 23, 477-484.

98. Zhang, W.; Yin, Z.H. Analysis of Volatile Constituents in Paederia scandens by HS-SPME-GC/MS. Chin. J. Exp. Tradit. Med. Formulae 2015, 21, 55-57. [CrossRef]

99. Geng, G.D.; Zhang, S.Q.; Cheng, Z.H. Allelopathy and Allelochemicals of Root Exudates in Hot Pepper. Acta Hortic. Sin. 2009, 36, 873-878. [CrossRef]

100. Deng, J.; Zhang, S.; Zhang, F.; Zhang, Y.; Hu, F.; Li, H. Autotoxins exuded from roots and the effects of PAEs on antioxidant capacity in roots of tobacco seedlings. Acta Ecol. Sin. 2017, 37, 495-504.

101. Yang, G.; Zhou, B.; Zhang, X.; Zhang, Z.; Wu, Y.; Zhang, Y.; Lu, S.; Zou, Q.; Gao, Y.; Teng, L. Effects of Tomato Root Exudates on Meloidogyne incognita. PLoS ONE 2016, 11, e0154675. [CrossRef]

102. Zhang, Z. Chemical Constituents and Antibacterial Activity of Essential Oil from Saxifraga stolonifera. Acta Agric. Boreali-Occident. Sin. 2016, 25, 1536-1540.

103. Firouzi, J.; Gohari, A.R.; Rustaiyan, A.; Larijani, K.; Saeidnia, S. Composition of the Essential oil of Nizamuddinia zanardinii, a Brown Alga Collected from Oman Gulf. J. Essent. Oil Bear. Plants. 2013, 16, 689-692. [CrossRef]

104. Monrroy, M.; Arauz, O.; Garcia, J.R. Active Compound Identification in Extracts of N. lappaceum Peel and Evaluation of Antioxidant Capacity. J. Chem. 2020, 2020, 4301891. [CrossRef] 
105. Zhang, S.; Xia, W.; Yang, X.; Zhang, T. Inhibition effect on Microcystis aeruginosa PCC7806 as well as separation and identification of algicidal substances isolated from Salvinia natans (L.) All. J. Hyg. Res. 2016, 45, 442-447. [CrossRef]

106. Zhao, L.; Yang, X.; Yang, Y.; Li, S.; Wang, H.; Wang, Y.; Zhang, S. Chemical Components and Antitumor Activity of Root Extract of Stellera chamaejasme L. Nat. Prod. Res. Dev. 2018, 30, 621-628. [CrossRef]

107. Aboobaker, Z.; Viljoen, A.; Chen, W.; Crous, P.W.; Maharaj, V.J.; Van, V.S. Endophytic fungi isolated from Pelargonium sidoides DC: Antimicrobial interaction and isolation of a bioactive compound. S. Afr. J. Bot. 2019, 122, 535-542. [CrossRef]

108. Rajamanikyam, M.; Vadlapudi, V.; Parvathaneni, S.P.; Koude, D.; Sripadi, P.; Misra, S.; Amanchy, R.; Upadhyayula, S.M. Isolation and characterization of phthalates from Brevibacterium mcbrellneri that cause cytotoxicity and cell cycle arrest. EXCLI J. 2017, 16, 375-387. [CrossRef]

109. Lee, D.S. Dibutyl phthalate, an alpha-glucosidase inhibitor from Streptomyces melanosporofaciens. J. Biosci. Bioeng. 2000, 89, 271-273. [CrossRef]

110. Al-Bari, M.A.A.; Bhuiyan, M.S.A.; Flores, M.E.; Petrosyan, P.; Garcia-Varela, M.; Ul Islam, M.A. Streptomyces bangladeshensis sp nov., isolated from soil, which produces bis-(2-ethylhexyl)phthalate. Int. J. Syst. Evol. Microbiol. 2005, 55, 1973-1977. [CrossRef]

111. Amade, P.; Mallea, M.; Bouaicha, N. Isolation, structural identification and biological activity of two metabolites produced by Penicillium olsonii Bainier and Sartory. J. Antibiot. 1994, 47, 201-207. [CrossRef] [PubMed]

112. Roy, R.N.; Laskar, S.; Sen, S.K. Dibutyl phthalate, the bioactive compound produced by Streptomyces albidoflavus 321.2. Microbiol. Res. 2006, 161, 121-126. [CrossRef] [PubMed]

113. Callaway, R.M.; Aschehoug, E.T. Invasive plants versus their new and old neighbors: A mechanism for exotic invasion. Science 2000, 290, 521-523. [CrossRef] [PubMed]

114. Shao, H.; Huang, X.; Wei, X.; Zhang, C. Phytotoxic effects and a phytotoxin from the invasive plant Xanthium italicum Moretti. Molecules 2012, 17, 4037-4046. [CrossRef]

115. Xuan, T.D.; Chung, I.M.; Khanh, T.D.; Tawata, S. Identification of phytotoxic substances from early growth of barnyard grass (Echinochloa crusgalli) root exudates. J. Chem. Ecol. 2006, 32, 895-906. [CrossRef]

116. Huang, X.; Li, Y.; Jiang, P.; Zhang, X.; Zhang, X.; Tan, P.; Tian, W. Identification of chrysanthemum root exudates and allelopathic effects of the three plants. Hubei Agric. Sci 2017, 56, 1061-1071. [CrossRef]

117. Deng, J.; Zhang, Y.; Hu, J.; Jiao, J.; Hu, F.; Li, H.; Zhang, S. Autotoxicity of Phthalate Esters in Tobacco Root Exudates: Effects on Seed Germination and Seedling Growth. Pedosphere 2017, 27, 1073-1082. [CrossRef]

118. Atanasov, A.G.; Waltenberger, B.; Pferschy-Wenzig, E.M.; Linder, T.; Wawrosch, C.; Uhrin, P.; Temml, V.; Wang, L.; Schwaiger, S.; Heiss, E.H.; et al. Discovery and resupply of pharmacologically active plant-derived natural products: A review. Biotechnol. Adv. 2015, 33, 1582-1614. [CrossRef]

119. Habib, M.R.; Karim, M.R. Antimicrobial and Cytotoxic Activity of Di-(2-ethylhexyl) Phthalate and Anhydrosophoradiol-3-acetate Isolated from Calotropis gigantea (Linn.) Flower. Mycobiology 2009, 37, 31-36. [CrossRef]

120. El-Mehalawy, A.; Gebreel, H.; Rifaat, H.; El-Kholy, I.; Humid, A. Effect of antifungal compounds produced by certain bacteria on physiological activities of human and plant pathogenic fungi. J. Appl. Sci. Res. 2008, 4, 425-432.

121. Li, X.; Jing, T.; Zhou, D.; Zhang, M.; Qi, D.; Zang, X.; Zhao, Y.; Li, K.; Tang, W.; Chen, Y.; et al. Biocontrol efficacy and possible mechanism of Streptomyces sp. H4 against postharvest anthracnose caused by Colletotrichum fragariae on strawberry fruit. Postharvest Biol. Technol. 2021, 175, 111401. [CrossRef]

122. Qi, G.; Liu, H.; Li, Z.; Long, L.; Wang, M. Active constituents of garlic bulb extract and its inhibition on Colletotrichum gloeosporioides. J. Henan Agri. Uni. 2020, 54, 59-63.

123. Zhang, Z.; Ao, W.; Xiong, Y.; Yan, R.; Wang, Y.; Zhu, D. Identification of antagonistic endophytic actinomycete FRo2 and isolation of its antimicrobial composition. Microbiology China. 2014, 41, 1574-1581. [CrossRef]

124. Liang, C.; Chen, Y.; Li, C.; Song, X.; Xie, C.; Zhu, C.; Sun, R. Antifungal activity of extracts of Helicteres ngustifolia against ten plant pathogenic fungi. Guihaia 2020, 40, 715-726.

125. Janu, N.P.; Jaynthy, C. Antimicrobial activity of diethyl phthalate: An insilico approach. Asian J. Pharm. Clin. Res. 2014, 7, 141-142.

126. Gayatri, K.V.; Soundhari, C.; Pavithra, B.P. Biofilm inhibitory effect of Chlorella extracts on Pseudomonas aeruginosa. Int. J. Pharm. Sci. Res. 2019, 10, 1966-1971. [CrossRef]

127. Rashiya, N.; Padmini, N.; Ajilda, A.A.K.; Prabakaran, P.; Durgadevi, R.; Ravi, A.V.; Ghosh, S.; Sivakumar, N.; Selvakumar, G. Inhibition of biofilm formation and quorum sensing mediated virulence in Pseudomonas aeruginosa by marine sponge symbiont Brevibacterium casei strain Alu 1. Microb. Pathog. 2021, 150, 104693. [CrossRef]

128. Wang, Z.; Wang, C.; You, Y.; Xu, W.; Lv, Z.; Liu, Z.; Chen, W.; Shi, Y.; Wang, J. Response of Pseudomonas fluorescens to dimethyl phthalate. Ecotoxicol. Environ. Saf. 2019, 167, 36-43. [CrossRef] [PubMed]

129. Xu, H.; He, X.Q. Natural products-based insecticidal agents 6. Design, semisynthesis, and insecticidal activity of novel monomethyl phthalate derivatives of podophyllotoxin against Mythimna separata Walker in vivo. Bioorg. Med. Chem. Lett. 2010, 20, 4503-4506. [CrossRef]

130. Brown, M.; Hebert, A.A. Insect repellents: An overview. J. Am. Acad. Dermatol. 1997, 36, 243-249. [CrossRef]

131. Guo, Y.; Kannan, K. Challenges encountered in the analysis of phthalate esters in foodstuffs and other biological matrices. Anal. Bioanal. Chem. 2012, 404, 2539-2554. [CrossRef]

132. Guo, Y.; Wang, L.; Kannan, K. Phthalates and parabens in personal care products from China: Concentrations and human exposure. Arch. Environ. Contam. Toxicol. 2014, 66, 113-119. [CrossRef] 
133. He, M.; Yang, C.; Geng, R.J.; Zhao, X.G.; Hong, L.; Piao, X.F.; Chen, T.; Quinto, M.; Li, D.H. Monitoring of phthalates in foodstuffs using gas purge microsyringe extraction coupled with GC-MS. Anal. Chim. Acta 2015, 879, 63-68. [CrossRef]

134. Chen, C.Y. Biosynthesis of di-(2-ethylhexyl) phthalate (DEHP) and di- $n$-butyl phthalate (DBP) from red alga-Bangia atropurpurea . Water Res. 2004, 38, 1014-1018. [CrossRef]

135. Namikoshi, M.; Fujiwara, T.; Nishikawa, T.; Ukai, K. Natural abundance 14C content of dibutyl phthalate (DBP) from three marine algae. Mar. Drugs 2006, 4, 290-297. [CrossRef]

136. Tian, C.; Ni, J.; Chang, F.; Liu, S.; Xu, N.; Sun, W.; Xie, Y.; Guo, Y.; Ma, Y.; Yang, Z.; et al. Bio-Source of di- $n$-butyl phthalate production by filamentous fungi. Sci. Rep. 2016, 6, 19791. [CrossRef] [PubMed]

137. Khatiwora, E.; Adsul, V.B.; Pawar, P.; Joseph, M.; Deshpande, N.R.; Kashalkar, R.V. Larvicidal activity of Ipomoea carnea stem extracts and its active ingredient dibutyl phthalate against Aedes aegypti and Culex quinquefasciatus. Pharma Chem. 2014, 6, 155-161.

138. Babu, B.; Wu, J.T. Production of phthalate esters by nuisance freshwater algae and cyanobacteria. Sci. Total Environ. 2010, 408, 4969-4975. [CrossRef] [PubMed]

139. Sun, H.; Wang, Y. Potential allelopathic effects of allelochemicals in aqueous extracts of leaves and root exudates of Capsicum annuum on vegetable crops. Allelopath. J. 2015, 35, 11-22.

140. Ludwig, M.J. Plants and endophytes: Equal partners in secondary metabolite production? Biotechnol. Lett. 2015, 37, 1325-1334. [CrossRef] 